\title{
Synthesis, Characterization and Antimicrobial Activity of Novel Chalcones Analogues
}

\author{
BUSHRA AHMED KATEB ${ }^{1,2}$, ABDULKAREEM ALI HUSSIEN ${ }^{1,2}$, \\ P. A. KULKARNI ${ }^{1}$, M. A. BASEER ${ }^{1}$
}

${ }^{1}$ P. G. Research Center, Department of Chemistry, Yeshwant Mahavidyalaya, Nanded-431602 (MS), India

${ }^{2}$ Hodiedah University, Education College, Yemen

nice1422@gmail.com

Received 14 January 2016 / Accepted 13 February 2016

\begin{abstract}
A new series of chalcones (3a-j) have been prepared by Claisen-Schmidt condensation between substituted acetophenone and substituted aldehyde. All these compounds were characterized by physical and spectral methods such as melting point, IR, ${ }^{1} \mathrm{H}$ NMR and Mass analysis. All the synthesized compounds have been screened for their antimicrobial activity.
\end{abstract}

Keywords: Chalcones, Spectral analysis, Anti microbial Activity

\section{Introduction}

Chalcones are a major class of natural products belonging to the flavonoid family. They are considered as the precursors of flavonoids and isoflavonoids. They are also the precursors of a number of biologically important heterocycles such as benzothiazepines, pyrazolines and flavones ${ }^{1}$.

Chalcones (trans-1, 3-diaryl-2propen-1-ones) are $\alpha, \beta$-unsaturated ketones consisting of two aromatic rings having diverse array of substituents. Rings are interconnected by a highly electrophonic three carbon $\alpha, \beta$-unsaturated carbonyl system that assumes linear or nearly planar structure ${ }^{2-4}$. They contain the electron ketoethylenic group $(-\mathrm{CO}-\mathrm{CH}=\mathrm{CH}-)$. Chalcones possess conjugated double bonds and a completely delocalized $\pi$ - system on both benzene rings. Chalcones have been used as intermediate for the preparation of compounds having therapeutic value ${ }^{5-7}$. Chalcones and their derivatives, whether synthetic or naturally occurring are an interesting and significant group of molecules as they possess a wide range of pharmacological activities such as anti-inflammatory, antimicrobial, antifungal, antibacterial, antioxidant, cytotoxic antitumor, anticancer, antimitotic, antileishmanial, antimalarial, antitubercular, antiviral and so on ${ }^{8-21}$. 


\section{Claisen-Schmidt condensation}

\section{Synthetic method of preparing chalcones}

The most convenient method is the Claisen Schimdt condensation of equimolar quantities of aryl methyl ketone with aryl aldehyde in the presence of alcoholic alkali ${ }^{22}$.

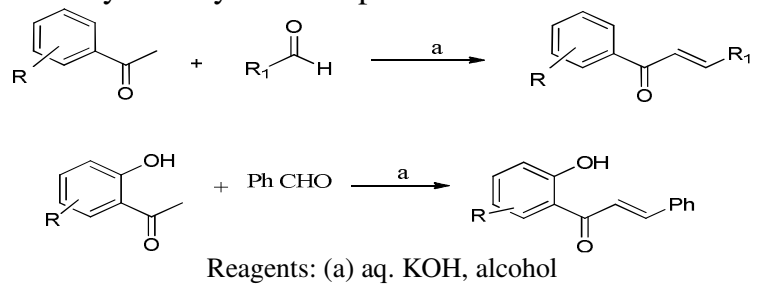

\section{Scheme 1}

The synthesis of chalcone compounds incorporating with hetero cycles became great importance in medicinal chemistry ${ }^{23-24}$. The hetero atoms in ther structure such as $(\mathrm{S}, \mathrm{N}, \mathrm{O})$ explain variety applications in the biological engineering and in other field of their specific structures ${ }^{25}$.

\section{Experimental}

Melting points of the compounds were determined in open capillary tubes and are uncorrected, IR Spectra were recorded on Shimadzu FT-IR Spectrometer using potassium bromide pellets, ${ }^{1} \mathrm{H}$ NMR was determined on a Bruker Avance II 400 Spectrometer against TMS as internal standard. Mass spectra were recorded on waters Micromass Q-T of Micro spectrometry.

General method for the synthesis of chalcones

A mixture of substituted acetophenone $(1 \mathrm{mmol})$, substituted aldehyde $(1 \mathrm{mmol})$ and $\mathrm{KOH}$ ( $2 \mathrm{mmol}$, in minium $\mathrm{H}_{2} \mathrm{O}$ ) were taken in ethanol and stirred at $50-60{ }^{\circ} \mathrm{C}$ temperature for one hour. The completion of reaction was monitored by TLC. The products were isolated by acidification of the cool diluted acid solution and obtained solid product was filtered and washed with water and recrystallize by ethanol to get pure product.<smiles>[R]Cc1c([R4])c([R4])cc(C(C)=O)c1[R4]</smiles>

1

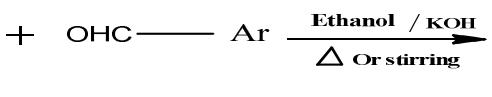

2

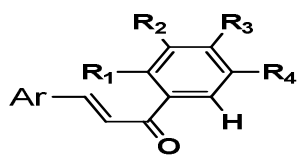

Chalcone $(3 a-j)$

Scheme 1. Synthesis of chalcones

Table 1. Substituted Acetophenones

\begin{tabular}{ccccccc}
\hline Comp. No & Product & $\mathrm{R}_{1}$ & $\mathrm{R}_{2}$ & $\mathrm{R}_{3}$ & $\mathrm{R}_{4}$ & $\mathrm{Ar}^{*}$ \\
\hline 1 & $\mathbf{3 a}$ & $\mathrm{H}$ & $\mathrm{I}$ & $\mathrm{OH}$ & $\mathrm{I}$ & $\mathrm{Ar}_{1}$ \\
2 & $\mathbf{3 b}$ & $\mathrm{OH}$ & $\mathrm{I}$ & $\mathrm{H}$ & $\mathrm{I}$ & $\mathrm{Ar}_{1}$ \\
3 & $\mathbf{3 c}$ & $\mathrm{OH}$ & $\mathrm{I}$ & $\mathrm{H}$ & $\mathrm{Cl}$ & $\mathrm{Ar}_{1}$ \\
4 & $\mathbf{3 d}$ & $\mathrm{OH}$ & $\mathrm{I}$ & $\mathrm{H}$ & $\mathrm{CH}_{3}$ & $\mathrm{Ar}_{1}$ \\
5 & $\mathbf{3 e}$ & $\mathrm{H}$ & $\mathrm{I}$ & $\mathrm{OH}$ & $\mathrm{I}$ & $\mathrm{Ar}_{2}$ \\
6 & $\mathbf{3 f}$ & $\mathrm{OH}$ & $\mathrm{I}$ & $\mathrm{H}$ & $\mathrm{I}$ & $\mathrm{Ar}_{2}$ \\
7 & $\mathbf{3 g}$ & $\mathrm{OH}$ & $\mathrm{I}$ & $\mathrm{H}$ & $\mathrm{Cl}$ & $\mathrm{Ar}_{2}$ \\
8 & $\mathbf{3 h}$ & $\mathrm{OH}$ & $\mathrm{I}$ & $\mathrm{H}$ & $\mathrm{CH}_{3}$ & $\mathrm{Ar}_{2}$ \\
9 & $\mathbf{3 i}$ & $\mathrm{H}$ & $\mathrm{I}$ & $\mathrm{OH}$ & $\mathrm{I}$ & $\mathrm{Ar}_{3}$ \\
10 & $\mathbf{3 j}$ & $\mathrm{OH}$ & $\mathrm{Br}$ & $\mathrm{H}$ & $\mathrm{Cl}$ & $\mathrm{Ar}_{4}$ \\
\hline
\end{tabular}


<smiles>[Y]c1ccc(Br)s1</smiles>

$\mathrm{Ar}_{1}$

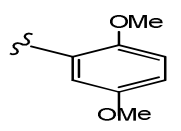

$\mathrm{Ar}_{2}$<smiles>[Li]c1c[nH]c2ccccc12</smiles>

$\mathrm{Ar}_{3}$<smiles>Cc1ccc(O)cc1</smiles>

$\mathrm{Ar}_{4}$

\section{Results and Discussion}

A variety of novel chalcones were synthesized via Claisen-Schmidt condensation of substituted acetophenones and aromatic benzaldehyde (Table 1). The reaction proceeded at room temperature. Work up procedure is simple and yield of the product is excellent.

All the newly synthesized chalcones were characterized by their chemical, physical and spectral analysis data (Table 2) and are further subjected to antimicrobial studies which exhibit moderate to good activity.

Table 2. Physical data of synthesized chalcones

\begin{tabular}{cccccc}
\hline Compd. No. & Product & Mol. Formula & Yield \% & M.P., ${ }^{\circ} \mathrm{C}$ & Solubility \\
\hline 1 & 3a & $\mathrm{C}_{13} \mathrm{H}_{7} \mathrm{O}_{2} \mathrm{I}_{2} \mathrm{BrS}$ & 90 & $176-178$ & DMF \\
2 & $\mathbf{3 b}$ & $\mathrm{C}_{13} \mathrm{H}_{7} \mathrm{O}_{2} \mathrm{I}_{2} \mathrm{BrS}$ & 92 & 162 & DMF \\
3 & $\mathbf{3 c}$ & $\mathrm{C}_{13} \mathrm{H}_{7} \mathrm{O}_{2} \mathrm{ClIBrS}$ & 80 & 178 & DMF \\
4 & $\mathbf{3 d}$ & $\mathrm{C}_{14} \mathrm{H}_{10} \mathrm{O}_{2} \mathrm{IbrS}$ & 92 & 142 & DMF \\
5 & $\mathbf{3 e}$ & $\mathrm{C}_{17} \mathrm{H}_{14} \mathrm{I}_{2} \mathrm{O}_{4}$ & 86 & $162-164$ & DMF \\
6 & $\mathbf{3 f}$ & $\mathrm{C}_{17} \mathrm{H}_{14} \mathrm{I}_{2} \mathrm{O}_{4}$ & 88 & $174-176$ & DMF \\
7 & $\mathbf{3 g}$ & $\mathrm{C}_{17} \mathrm{H}_{14} \mathrm{ClIO}_{4}$ & 80 & 178 & DMF \\
8 & $\mathbf{3 h}$ & $\mathrm{C}_{18} \mathrm{H}_{17} \mathrm{IO}_{4}$ & 90 & 159 & DMF \\
9 & $\mathbf{3 i}$ & $\mathrm{C}_{17} \mathrm{H}_{11} \mathrm{NI}_{2} \mathrm{O}_{2}$ & 88 & 150 & DMF \\
10 & $\mathbf{3 j}$ & $\mathrm{C}_{15} \mathrm{H}_{10} \mathrm{BrClO}_{3}$ & 85 & $108-110$ & DMF \\
\hline
\end{tabular}

\section{Spectral analysis of the compounds}

The structure of the compounds were done by spectral analysis (IR, ${ }^{1} \mathrm{H}$ NMR, Mass) and the results are shown below

\section{Compound $3 a$}<smiles>[2H]/C(=C(/C)C(=O)c1c(C)c(I)c(O)c(I)c1C)c1sc(Br)c(C)c1C</smiles>

(E)-3-(5-Bromothiophen-2-yl)-1-(4-hydroxy-3,5-diiodophenyl)prop-2-en-1-one

FTIR $\left(\mathrm{KBr}, \mathrm{cm}^{-1}\right)$ : 3224(OH), 1646(C=O), 1576(C=C), 1465(C-C Aromatic str), 683(C-Br). ${ }^{1} \mathrm{H}$ NMR: 7.24(d, $\left.1 \mathrm{H}, \mathrm{H}_{3}\right), 7.48\left(\mathrm{~d}, 1 \mathrm{H}, \mathrm{H}_{4}\right), 7.49(\mathrm{~d}, 1 \mathrm{H}, \mathrm{H \alpha}, \mathrm{J}=15 \mathrm{~Hz}), 7.58(\mathrm{~d}, 1 \mathrm{H}, \mathrm{H}$ $\beta, \mathrm{J}=15 \mathrm{~Hz}), 7.48\left(\mathrm{~s} 1 \mathrm{H}, \mathrm{H}_{5}\right), 7.53\left(\mathrm{~s}, 1 \mathrm{H}, \mathrm{H}_{6}\right), 8.43(\mathrm{~s}, 1 \mathrm{H}, \mathrm{OH})$.

M.S. $(\mathrm{m} / \mathrm{z}):(\mathrm{M})=560(\mathrm{~m}), 561(\mathrm{~m}+1), 558(\mathrm{~m}-2)$.

\section{Compound $\mathbf{3 b}$}<smiles>CC(=Cc1sc(Br)c(C)c1C)C(=O)c1c(C)c(I)c(C)c(I)c1O</smiles>

(E)-3-(5-Bromothiophen-2-yl)-1-(2-hydroxy-3,5-diiodophenyl)prop-2-en-1-one 
FTIR $\left(\mathrm{KBr}, \mathrm{cm}^{-1}\right)$ : 3425(OH), 1623(C=O), 1546(C=C), 1419(C-C Aromatic str), 621(C-Br). ${ }^{1} \mathrm{H}$ NMR: 7.33(d, $\left.1 \mathrm{H}, \mathrm{H}_{3}\right), 7.48\left(\mathrm{~d}, 1 \mathrm{H}, \mathrm{H}_{4}\right), 7.73(\mathrm{~d}, 1 \mathrm{H}, \mathrm{H \alpha}, \mathrm{J}=15 \mathrm{~Hz}), 7.97(\mathrm{~d}, 1 \mathrm{H}, \mathrm{H} \beta$, $\mathrm{J}=15 \mathrm{~Hz}), 8.27\left(\mathrm{~s}, 1 \mathrm{H}, \mathrm{H}_{5}\right), 8.55\left(\mathrm{~s}, 1 \mathrm{H}, \mathrm{H}_{6}\right), 13.7(\mathrm{~s}, 1 \mathrm{H}, \mathrm{OH})$. M.S. $(\mathrm{m} / \mathrm{z}):(\mathrm{M})=558(\mathrm{~m}-2)$.

\section{Compound $3 c$}<smiles>CC(C(=O)c1c(C)c(Cl)c(I)c(I)c1O)=C(C)c1sc(Br)c(C)c1C</smiles>

(E)-3-(5-Bromothiophen-2-yl)-1-(5-hydroxy-3-iodophenyl)prop-2-en-1-one

FTIR (KBr, cm $\left.{ }^{-1}\right)$ : 3436(OH), 1635(C=O), 1526(C=C), 1419(C-C Aromatic str), 621(C-Br). 802(C-Cl), 675(C-Br). ${ }^{1} \mathrm{H}$ NMR: 7.12(d, $\left.1 \mathrm{H}, \mathrm{H}_{3}\right), 7.21\left(\mathrm{~d}, 1 \mathrm{H}, \mathrm{H}_{4}\right), 7.27(\mathrm{~d}, 1 \mathrm{H}, \mathrm{H \alpha}, \mathrm{J}=15 \mathrm{~Hz})$, 7.97(d, $1 \mathrm{H}, \mathrm{H} \beta, \mathrm{J}=15 \mathrm{~Hz}), 8.23\left(\mathrm{~s}, 1 \mathrm{H}, \mathrm{H}_{5}\right), 8.47\left(\mathrm{~s}, 1 \mathrm{H}, \mathrm{H}_{6}\right), 13.46(\mathrm{~s}, 1 \mathrm{H}, \mathrm{OH})$. M.S. $(\mathrm{m} / \mathrm{z}): 468(\mathrm{~m})$.

\section{Compound $\mathbf{3 d}$}

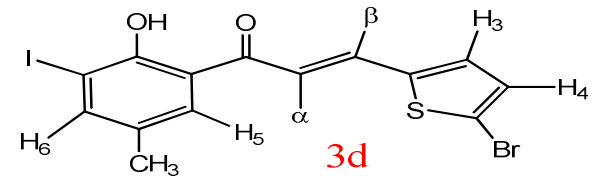

(E)-3-(5-Bromothiophen-2-yl)-1-(2-hydroxy-3-iodo-5-methylphenyl)prop-2-en-1-one FTIR $\left(\mathrm{KBr}, \mathrm{cm}^{-1}\right): 3425(\mathrm{OH}), 1639(\mathrm{C}=\mathrm{O}), 1569(\mathrm{C}=\mathrm{C}), 1415(\mathrm{C}-\mathrm{C}$ Aromatic str), 675(CBr).802(C-Cl), 675(C-Br). ${ }^{1} \mathrm{H}$ NMR: 2.32(s,3H,CH3), 7.27(d, $\left.1 \mathrm{H}, \mathrm{H}_{3}\right), \quad 7.52\left(\mathrm{~d}, 1 \mathrm{H}, \mathrm{H}_{4}\right)$, $7.65(\mathrm{~d}, 1 \mathrm{H}, \mathrm{H \alpha}, \mathrm{J}=15 \mathrm{~Hz}), 7.95(\mathrm{~d}, 1 \mathrm{H}, \mathrm{H} \quad \beta, \mathrm{J}=15 \mathrm{~Hz}), 7.85\left(\mathrm{~s}, 1 \mathrm{H}, \mathrm{H}_{5}\right), \quad 8.04\left(\mathrm{~s}, 1 \mathrm{H}, \mathrm{H}_{6}\right)$, 13.46(s,1H, OH). M.S. $(\mathrm{m} / \mathrm{z}): 450(\mathrm{~m}+1), 448(\mathrm{~m}-1), 447(\mathrm{~m}-2)$.

\section{Compound $3 \boldsymbol{e}$}<smiles>COc1c(C)c(C)c(/C(P)=C(\Cl)C(=O)c2cc(I)c(O)c(I)c2C)c(C)c1OC</smiles>

(E)-3-(2,5-Dimethoxyphenyl)-1-(4-hydroxy-3,5-diiiodophenyl)prop-2-en-1-one FTIR $\left(\mathrm{KBr}, \mathrm{cm}^{-1}\right): 3382(\mathrm{OH}), 1658(\mathrm{C}=\mathrm{O}), 1589(\mathrm{C}=\mathrm{C}), 1492\left(\mathrm{C}-\mathrm{C}\right.$ Aromatic str). ${ }^{1} \mathrm{HNMR}$ :

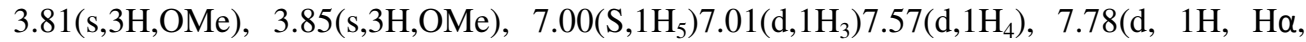
$\mathrm{J}=15 \mathrm{~Hz}), 8.00(\mathrm{~d}, 1 \mathrm{H}, \mathrm{H} \beta, \mathrm{J}=15 \mathrm{~Hz}), 8.45-8.50\left(\mathrm{~s}, 2 \mathrm{H}, \mathrm{H}_{6}, \mathrm{H}_{7}\right), 10.28(\mathrm{~s}, 1 \mathrm{H}, \mathrm{OH})$. M.S. $(\mathrm{m} / \mathrm{z})$ : 536(m), 535(m-1), 534(m-2).

\section{Compound $3 f$}<smiles></smiles>

(E)-3-(2,5-Dimethoxyphenyl)-1-(2-hydroxy-3,5-diiiodophenyl)prop-2-en-1-one 
FTIR $\left(\mathrm{KBr}, \mathrm{cm}^{-1}\right): 3433(\mathrm{OH}), 1635(\mathrm{C}=\mathrm{O}), 1558(\mathrm{C}=\mathrm{C}), 1431\left(\mathrm{C}-\mathrm{C}\right.$ Aromatic str). ${ }^{1}$ HNMR: 3.82(s,3H,OMe), 3.86(s,3H,Ome), 7.02( $\left(\mathrm{S}, 1 \mathrm{H}_{5}\right) 7.07\left(\mathrm{~d}, 1 \mathrm{H}_{3}\right) 7.46\left(\mathrm{~d}, 1 \mathrm{H}_{4}\right), 8.00(\mathrm{~d}, 1 \mathrm{H}, \mathrm{H \alpha}$, $\mathrm{J}=15 \mathrm{~Hz}), 8.22(\mathrm{~d}, 1 \mathrm{H}, \mathrm{H} \beta, \mathrm{J}=15 \mathrm{~Hz}), 8.23\left(\mathrm{~s}, 1 \mathrm{H}, \mathrm{H}_{6}\right), 8.57\left(\mathrm{~s}, 1 \mathrm{H}_{7}\right), 13.84(\mathrm{~s}, 1 \mathrm{H}, \mathrm{OH})$. M.S. $(\mathrm{m} / \mathrm{z}):$ 535(m-1), 534(m-2).

\section{Compound $\mathbf{3 g}$}<smiles></smiles>

(E)-1-(5-chloro-5hydroxy-3-iodophenyl)-3-(2,5-dimethoxyphenyl)prop-2-en-1-one

FTIR $\left(\mathrm{KBr}, \mathrm{cm}^{-1}\right): 3425(\mathrm{OH}), 1667(\mathrm{C}=\mathrm{O}), 1550(\mathrm{C}=\mathrm{C}), 1496(\mathrm{C}-\mathrm{C}$ Aromatic str). 785(C-Cl). ${ }^{1} \mathrm{HNMR}: 3.80(\mathrm{~s}, 3 \mathrm{H}, \mathrm{Ome}), 3.85(\mathrm{~s}, 3 \mathrm{H}, \mathrm{Ome}), 7.03\left(\mathrm{~S}, 1 \mathrm{H}_{5}\right) 7.22\left(\mathrm{~d}, 1 \mathrm{H}_{3}\right) 7.50\left(\mathrm{~d}, 1 \mathrm{H}_{4}\right), 8.04(\mathrm{~d}, 1 \mathrm{H}$, $\mathrm{H} \alpha, \mathrm{J}=15 \mathrm{~Hz}), 8.12(\mathrm{~d}, 1 \mathrm{H}, \mathrm{H} \beta, \mathrm{J}=15 \mathrm{~Hz}), 8.16\left(\mathrm{~s}, 1 \mathrm{H}, \mathrm{H}_{6}\right), 8.26\left(\mathrm{~s}, 1 \mathrm{H}_{7}\right), 13.51(\mathrm{~s}, 1 \mathrm{H}, \mathrm{OH})$. M.S. $(\mathrm{m} / \mathrm{z}): 443(\mathrm{~m}-1), 445(\mathrm{~m}+1)$.

\section{Compound $3 \mathrm{~h}$}<smiles></smiles>

(E)-3-(2,5-dimethoxyphenyl)-1-(2-hydroxy-3-iodo-5-methylphenyl)prop-2-en-1-one FTIR $\left(\mathrm{KBr}, \mathrm{cm}^{-1}\right): 3440(\mathrm{OH}), 1631(\mathrm{C}=\mathrm{O}), 1562(\mathrm{C}=\mathrm{C}), 1430\left(\mathrm{C}-\mathrm{C}\right.$ Aromatic str). ${ }^{1} \mathrm{H}$ NMR: 3.80(s,3H,Ome),3.85(s,3H,Ome), 7.03(S,1 $\left.\mathrm{H}_{5}\right) 7.22\left(\mathrm{~d}, 1 \mathrm{H}_{3}\right) 7.50\left(\mathrm{~d}, 1 \mathrm{H}_{4}\right), 8.04(\mathrm{~d}, 1 \mathrm{H}, \mathrm{H \alpha}, \mathrm{J}=15 \mathrm{~Hz})$, 8.12(d, $1 \mathrm{H}, \mathrm{H} \beta, \mathrm{J}=15 \mathrm{~Hz}), 8.16\left(\mathrm{~s}, 1 \mathrm{H}, \mathrm{H}_{6}\right), 8.26\left(\mathrm{~s}, 1 \mathrm{H}_{7}\right), 13.51(\mathrm{~s}, 1 \mathrm{H}, \mathrm{OH})$. M.S. $(\mathrm{m} / \mathrm{z}): 423(\mathrm{~m}-1)$.

\section{Compound $3 i$}<smiles>Cc1[nH]c2ccccc2c1C(C)C(=O)c1c(C)c(I)c(O)c(I)c1I</smiles>

(E)-1-(4- Hydroxy-3,5-diiodophenyl)-3-(1H-indol-3-yl)prop-2-en-1-one

FTIR $\left(\mathrm{KBr}, \mathrm{cm}^{-1}\right): 1663(\mathrm{C}=\mathrm{O}), 1570(\mathrm{C}=\mathrm{C}), 1461\left(\mathrm{C}-\mathrm{C}\right.$ Aromatic str). ${ }^{1} \mathrm{H}$ NMR: $7.18((\mathrm{~d}, 1 \mathrm{H}$, $\mathrm{H} \alpha, \mathrm{J}=15 \mathrm{~Hz}), 7.26(\mathrm{~d}, 1 \mathrm{H}, \mathrm{H} \quad \beta, \mathrm{J}=15 \mathrm{~Hz}), 8.05\left(\mathrm{~s}, 1 \mathrm{H}, \mathrm{H}_{3}\right), 8.12\left(\mathrm{~s}, 1 \mathrm{H}, \mathrm{H}_{4}\right), 8.14\left(\mathrm{~S}, 1 \mathrm{H}, \mathrm{H}_{5}\right), 8$ 8.45(m,4H,Ar-H) ,9. 95(s,1H, OH), 11.99(s,1H,NH) .M.S. $(\mathrm{m} / \mathrm{z}): 423(\mathrm{~m}-1)$.

\section{Compound $3 j$}

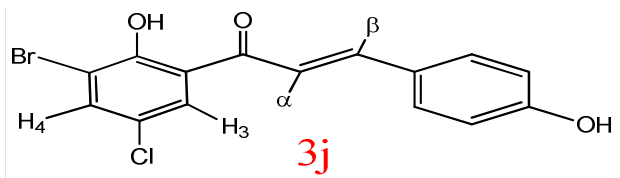

(E)-1-(3-Bromo-5-chloro-2- hydroxyphenyl)-3-(4- hydroxyphenyl)prop-2-en-1-one 
FTIR $\left(\mathrm{KBr}, \mathrm{cm}^{-1}\right)$ : 1641(C=O), 1563(C=C), 1422(C-C Aromatic str). ${ }^{1} \mathrm{H}$ NMR: 7.40((d, 1H, $\mathrm{H} \alpha, \mathrm{J}=15 \mathrm{~Hz}), \quad 8.51(\mathrm{~d}, \quad 1 \mathrm{H}, \quad \mathrm{H} \quad \beta, \mathrm{J}=15 \mathrm{~Hz}), \quad 7.44-7.77(\mathrm{~m}, 4 \mathrm{H}, \mathrm{Ar}-\mathrm{H}), \quad 9.95(\mathrm{~s}, 1 \mathrm{H}, \quad \mathrm{OH})$, 7.61(s,1H, $\left.\mathrm{H}_{3}, \mathrm{Ar}\right), 7.72\left(\mathrm{~s}, 1 \mathrm{H}, \mathrm{H}_{4}\right), \quad 11.99(\mathrm{~s}, 1 \mathrm{H}, \mathrm{NH}), \quad 8.24(\mathrm{~s}, 1 \mathrm{H}, \mathrm{OH}) . \quad$ M.S. $\quad(\mathrm{m} / \mathrm{z}): 353$ (M), $355(\mathrm{~m}+2), 351(\mathrm{~m}-2)$.

\section{Antimicrobial activity}

Antimicrobial screening was done using disc diffusion method ${ }^{26}$ at a concentration of $500 \mu \mathrm{g} / \mathrm{mL}$.

\section{Procedure}

The test was performed according to the disk diffusion method ${ }^{26}$ adopted with some modification for the prepared compound using Penciline and streptomycin as references. The prepared compounds were tested against one strain of Gram +ve bacteria, Gram -ve bactria, fungi. Whatman filter paper disk of $5 \mathrm{~mm}$ diameter were sterilized by autoclaving for $15 \mathrm{~min}$ at $121{ }^{\circ} \mathrm{C}$. The sterile disk were impregnated with different compounds ( $600 \mathrm{~g} / \mathrm{disk})$. Agar plates were surface inoculated uniformly from the both culture of the tested microorganism. The disk were placed on the medium suitably spaced apart on the plate were incubated at $50{ }^{\circ} \mathrm{C}$ for $1 \mathrm{~h}$ to permit good diffusion and then transferred to an incubator at $37{ }^{\circ} \mathrm{C}$ for $24 \mathrm{~h}$ for bacteria and $28{ }^{\circ} \mathrm{C}$ for $72 \mathrm{~h}$ for fungi.

The compounds were evaluated for antibacterial activity against Staphylococcus aureus gr $+\mathrm{ve}$, Escherichia coli gr -ve Bacillus subtilis gr +ve, Pseudomonas aeruginosa gr -ve, and antifungal activity against Aspergillus oryzoe, Aspergillus niger. DMSO was used as solvent control. The results of antimicrobial data are summarized in Table 3. All compounds show the moderate to good activity against bacteria, but for all compounds did not stop the growth of Aspergillus oryzoe fungus and all compounds were not effective towards Aspergillus niger fungus.

Table 3. Antimicrobial activity of synthesized compounds

\begin{tabular}{ccccccc}
\hline \multirow{2}{*}{ Compounds } & \multicolumn{2}{c}{$\begin{array}{c}\text { Gram positive } \\
\text { bacterias }\end{array}$} & \multicolumn{2}{c}{ Gram negative bacterias } & \multicolumn{2}{c}{ Fungus } \\
& Staph & Bacillus & Escherichia & Pseudomonas & Aspergillus & Aspergillus \\
& aureus & subtilis & coli & aeruginosa & oryzoe & niger \\
\hline 3a & ++ & ++ & $-\mathrm{ve}$ & ++ & $-\mathrm{ve}$ & - \\
$\mathbf{3 b}$ & - & - & $-\mathrm{ve}$ & $-\mathrm{ve}$ & $-\mathrm{ve}$ & - \\
$\mathbf{3 c}$ & - & - & $-\mathrm{ve}$ & $-\mathrm{ve}$ & $-\mathrm{ve}$ & - \\
$\mathbf{3 d}$ & - & - & $-\mathrm{ve}$ & $-\mathrm{ve}$ & $-\mathrm{ve}$ & - \\
$\mathbf{3 e}$ & ++ & ++ & $-\mathrm{ve}$ & ++ & $-\mathrm{ve}$ & - \\
$\mathbf{3 f}$ & - & - & $-\mathrm{ve}$ & $-\mathrm{ve}$ & $-\mathrm{ve}$ & - \\
$\mathbf{3 g}$ & - & - & $-\mathrm{ve}$ & + & $-\mathrm{ve}$ & - \\
$\mathbf{3 h}$ & - & - & $-\mathrm{ve}$ & $-\mathrm{ve}$ & $-\mathrm{ve}$ & - \\
$\mathbf{3 i}$ & ++ & ++ & + & ++ & $-\mathrm{ve}$ & - \\
$\mathbf{3 j}$ & ++ & + & $-\mathrm{ve}$ & + & $-\mathrm{ve}$ & - \\
Penciline 1 & + & + & + & + & $\mathrm{X}$ & $\mathrm{X}$ \\
Streptomycin 2 & ++ & ++ & ++ & ++ & $\mathrm{X}$ & $\mathrm{X}$ \\
\hline
\end{tabular}

$++=$ Clear Zone of Inhibition $+=$ Minimum Zone of Inhibition -ve = Growth (Antibacteria and Antifungul Activities Observed) - = No Effect $X=$ Not applicable, Standerd 1 Penciline + , Standerd 2 Streptomycin++ 


\section{Conclusion}

We have reported some novel chalcones using substituted acetophenone with substituted aldehyde with high yield. The newly synthesized chalcones were confirmed by spectral analysis and further evaluated for their antimicrobial activity. The antibacterial activity revealed that of the compounds showed moderate to good activity against the pathogens used, the result was negative on fungus.

\section{Acknowledgement}

The authors are thankful to Head of department of Chemistry, Head of department of Microbilogy, Principal of Yeshwant College, Nanded for providing lab facilities for the research work.

\section{References}

1. Rahman M A, Chem Sci J., 2011, 29, 1-16

2. Awasthi S K, Mishra N, Kumar B, Sharma M, Bhattacharya A, Mishra L C and Bhasin V K, Med Chem Res., 2009, 18(6), 407-420; DOI:10.1007/s00044-008-9137-9

3. Cheng M S, Shili R and Kenyon G, Chinese Chem Lett., 2000, 11(10), 851-854.

4. Lim S S, Kim H S and Lee D U, Bulletin Korean Chem Soc., 2007, 28(12), 24952497; DOI:10.5012/bkcs.2007.28.12.2495

5. Straub T S, Tetrahedron Lett., 1995, 36, 663-664; DOI:10.1016/00404039(94)02346-D

6. Sandler S and Karo W, In Organic Functional Group Preparations. 1972, 3, 372; DOI:10.1016/b978-0-08-092558-5.50004-6

7. Bergman E D, Ginsibm L and Pappo R, Org React., 1959, 10, 179; DOI:10.1002/0471264180.or010.03

8. Bandgar B P, Patil S A, Gacche R N, Korbad B L, Hote B S, Kinkar S N and Jalde S S, Bioorg Med Chem Lett., 2010, 20(2), 730-733; DOI:10.1016/j.bmcl.2009.11.068

9. Cabrera M, Simoens M, Falchi G, Lavaggi ML, Piro O E, Castellano E E, Vidal A, Azqueta A, Monge A, Cerain AL, Sagrera G, Seoane G, Cerecettoa H and Gonzaleza M, Bioorg Med Chem., 2007, 15(10), 3356-3367; DOI:10.1016/j.bmc.2007.03.031

10. Patel A A and Mehta A G, J Saudi Chem Soc., 2010, 14(2), 203-208; DOI:10.1016/j.jscs.2010.02.012

11. Suryawanshi S N, Chandra N, Kumar P, Porwal J and Gupta S, Eur J Med Chem., 2008, 43(11), 2473-2478; DOI:10.1016/j.ejmech.2007.12.014

12. Hayat F, Moseley E, Salahuddin A, Zyl R L V and Azam A, Eur J Med Chem., 2011, 46(5), 1897-1905; DOI:10.1016/j.ejmech.2011.02.004

13. Kumar N, Chauhan A and Drabu S, Biomed Pharmacother., 2011, 65(5), 375-380; DOI:10.1016/j.biopha.2011.04.023

14. Agarwal A, Srivastava K, Purib S K, Chauhan P M S, Bioorg Med Chem., 2005, 13(15), 4645-4650; DOI:10.1016/j.bmc.2005.04.061

15. Bandgar B P, Gawande S S, Bodade R G, Gawande N M and Khobragade C N, Bioorg Med Chem., 2009, 17(24), 8168-8173; DOI:10.1016/j.bmc.2009.10.035

16. Bandgar B P and Gawande S S, Bioorg Med Chem., 2010, 18(5), 2060-2065; DOI:10.1016/j.bmc.2009.12.077

17. Sharma A, Chakravarti B, Gupta M P, Siddiqui J A, Konwar R and Tripathi R P, Bioorg Med Chem., 2010, 18(13), 4711-4720; DOI:10.1016/j.bmc.2009.12.077 
18. Insuasty B, Tigreros A, Orozco F, Quiroga J, Abonia R, Nogueras M, Sanchez A and Cobo J, Bioorg Med Chem., 2010, 18(14), 4965-4974;

DOI:10.1016/j.bmc.2010.06.013

19. Liaras K, Geronikaki A, Glamoclija J, Ciric A and Sokovic M, Bioorg Med Chem., 2011, 19(10), 3135-3140; DOI:10.1016/j.bmc.2011.04.007

20. Liaras K, Geronikaki A, Glamoclija J, Ciric A and Sokovic M, Bioorg Med Chem., 2011, 19(24), 7349-7356; DOI:10.1016/j.bmc.2011.10.059

21. Anand N, Singh P, Sharma A, Tiwari S, Singh V, Singh D K, Srivastava K K and Singh B N, Bioorg Med Chem., 2012, 20(17), 5150-5163;

DOI:10.1016/j.bmc.2012.07.009

22. Taylor E C and Morrison R W, J Org Chem., 1967, 32(8), 2379-2382; DOI:10.1021/jo01283a004

23. Padhy A K, Bardham M and Danda C S, Indian J Chem., 2003, 42B(4), 910-915.

24. Nakum K H and Shah V H, Indian J Het Chem., 2002, 12(1), 37.

25. Nagham M A J, Chem Chemi Sci., 2013, 3(2), 70-78.

26. Afaf H, El-masry H H, Fahmy and Ali S H, Abdelwahed, Molecules, 2000, 5(12), 1429-1438; DOI:10.3390/51201429 